\title{
Posterior Thoracic Cage Interbody Fusion Offers Solid Bone Fusion with Sagittal Alignment Preservation for De- compression and Fusion Surgery in Lower Thoracic and Thoracolumbar Spine
}

\author{
Hong Kyung Shin, ${ }^{1}$ Moinay Kim, Sun Kyu Oh, ${ }^{2}$ Il Choi, ${ }^{3}$ Dong Kwang Seo, Jin Hoon Park, Sung Woo Roh, Sang Ryong Jeon \\ Department of Neurological Surgery, Asan Medical Center, University of Ulsan College of Medicine, Seoul, Korea \\ Department of Neurological Surgery, ${ }^{2}$ National Police Hospital, Seoul, Korea \\ Department of Neurological Surgery, ${ }^{3}$ Dongtan Sacred Heart Hospital, College of Medicine, Hallym University, Hwaseong, Korea
}

Objective : It is challenging to make solid fusion by posterior screw fixation and laminectomy with posterolateral fusion (PLF) in thoracic and thoracolumbar (TL) diseases. In this study, we report our experience and follow-up results with a new surgical technique entitled posterior thoracic cage interbody fusion (PTCIF) for thoracic and TL spine in comparison with conventional PLF.

Methods : After institutional review board approval, a total of 57 patients who underwent PTCIF $(n=30)$ and conventional PLF $(n=27)$ for decompression and fusion in thoracic and TL spine between 2004 and 2019 were analyzed. Clinical outcomes and radiological parameters, including bone fusion, regional Cobb angle, and proximal junctional Cobb angle, were evaluated.

Results : In PTCIF and conventional PLF, the mean age was 61.2 and 58.2 years $(p=0.46)$, and the numbers of levels fused were 2.8 and 3.1 ( $p=0.46$ ), respectively. Every patient showed functional improvement except one case of PTCIF. Postoperative hematoma as a perioperative complication occurred in one and three cases, respectively. The mean difference in the regional Cobb angle immediately after surgery compared with that of the last follow-up was $1.4^{\circ}$ in PTCIF and $7.6^{\circ}$ in conventional PLF $(p=0.003)$, respectively. The mean durations of postoperative follow-up were 35.6 months in PTCIF and 37.3 months in conventional PLF $(p=0.86)$.

Conclusion : PTCIF is an effective fusion method in decompression and fixation surgery with good clinical outcomes for various spinal diseases in the thoracic and TL spine. It provides more stable bone fusion than conventional PLF by anterior column support.

Key Words : Decompression - Lumbar vertebrae · Posterior thoracic cage interbody fusion · Spinal fusion · Thoracic vertebrae.

\section{INTRODUCTION}

In lumbar spine, posterior lumbar interbody fusion (PLIF) with posterior screw fixation (PSF) using a cage packed with bone chips is widely used. A higher fusion rate, endurable axial loading and safety make it more popular among surgical op-

- Received : November 3, 2020 •Revised : December 4, 2020 •Accepted : March 19, 2021

- Address for reprints : Sang Ryong Jeon

Department of Neurological Surgery, Asan Medical Center, University of Ulsan College of Medicine, 88 Olympic-ro 43-gil, Songpa-gu, Seoul 05505, Korea Tel : +82-2-3010-3550, Fax : +82-2-476-6739, E-mail : srjeon@amc.seoul.kr, ORCID : https://orcid.org/0000-0002-8340-7978 
tions, rather than conventional posterolateral fusion (PLF) ${ }^{16,19)}$. As a result, PLIF has been widely employed in lumbar spinal lesion where decompressive laminectomy and bone fusion is mandatory.

On the contrary, in thoracic and thoracolumbar (TL) spine, because cage insertion to disc space is difficult, owing to the difficulty of dural sac retraction, interbody cage insertion has not been performed, and anterior approach and interbody fusion was developed with satisfactory results for thoracic spine $^{15)}$. However, limitations of anterior approach associated with diverse morbidities, especially pulmonary complications, exist as well ${ }^{14}$. In addition, in thoracic and TL spine, owing to the spinal cord exposure, posterolateral bone chip application for bone fusion is not easy, because of its risky probability of bone chip migration onto the spinal cord.

Therefore, we conducted posterior thoracic cage interbody fusion (PTCIF) in a similar method to PLIF technique for patients requiring posterior decompression and fusion in the thoracic and TL spine for traumatic injury or degenerative disease. and demonstrated the fusion rate and operation technique in the previous report ${ }^{10)}$. In the present study, to evaluate the results of PTCIF objectively, we retrospectively compared the clinical and radiological outcome of PTCIF to the conventional PLF in the thoracic and TL spine.

\section{MATERIALS AND METHODS}

\section{Patient identification}

After Institutional Review Board (IRB) of Asan Medical Center (2019-1116) approval, we retrospectively reviewed the medical records of the patients who underwent thoracic or TL spinal fusion surgery between April 2004 and April 2019. Inclusion criteria were burst fracture, fracture-dislocation, traumatic compression fracture, spinal stenosis, spondylolisthesis, and herniated disc. Initially, a total of 123 patients were enrolled : PTCIF was consecutively performed on 30 patients and conventional PLF was performed on 93 patients. In conventional PLF, 66 patients were excluded due to fusion without decompression (46 patients), short follow-up less than 6 months (15 patients), fusion above T4-5 level (three patients), and adjacent segmental disease (ASD; two patients). Finally, a total of 57 patients ( 30 patients who underwent PTCIF and 27 patients who underwent conventional PLF) were analyzed on this study (Fig. 1). The IRB waives the requirement of obtaining informed consent for this study.

\section{Surgical procedures}

All surgeries were performed by a single surgeon (S.R.J.). Before surgery, magnetic resonance imaging and computed tomography (CT) were performed to all patients to diagnose and determine the levels of surgery. Intraoperative monitoring standards, including a somatosensory and motor evoked potential, and electromyography, were monitored. Serial plain X-

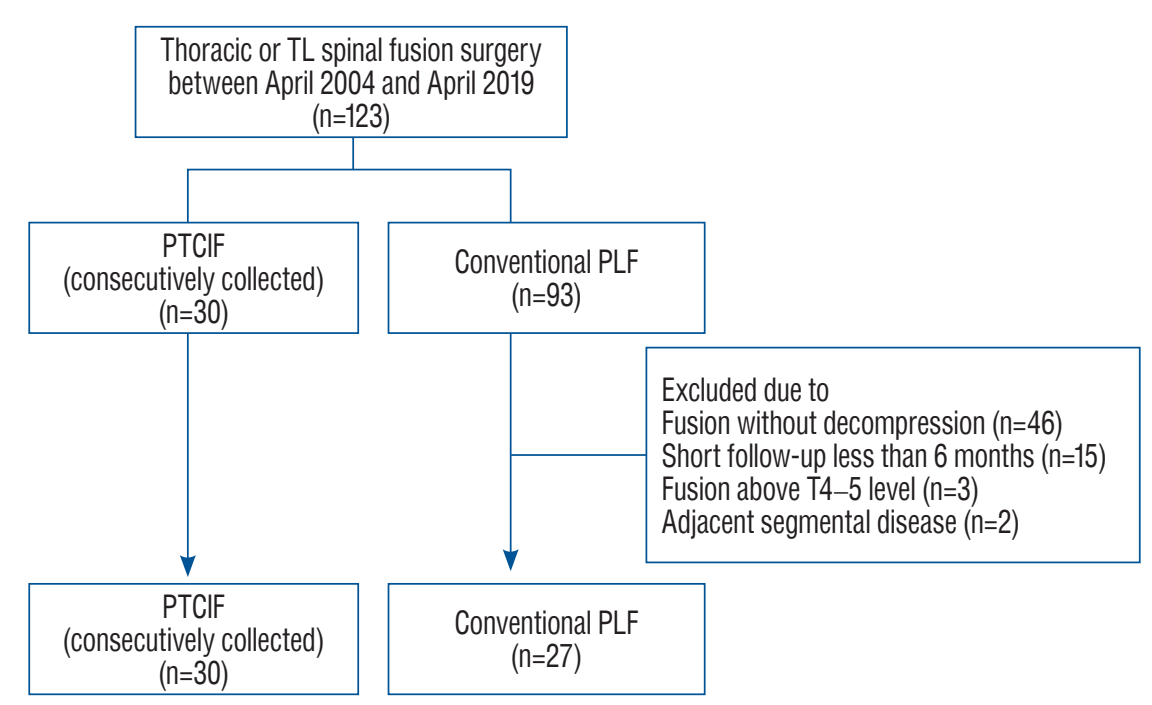

Fig. 1. A flowchart of the study. TL : thoracolumbar, PTCIF : posterior thoracic cage interbody fusion, PLF : posterolateral fusion. 
rays were performed after the operation and subsequently at regular follow-ups to evaluate the accuracy of instrumentation and maintenance of alignment. In PTCIF, thin-section CT with sagittal and coronal reconstruction was used to evaluate the bone fusion rates at 3 months for 1 level PTCIF, and 4 months for more than 2 levels. In conventional PLF, simple spine X-rays were evaluated 1, 3, 6, and 12 months after operation. If patients experienced any associated symptoms, more regular visits were recommended.

\section{PTCIF technique}

In the previous report, the authors described surgical technique of PTCIF including video demonstration ${ }^{10)}$. To briefly describe it, the PTCIF technique is as follows. Under general anesthesia, the patient lays in the prone position on the spine table (Jackson Spine Table; Mizuho OSI, Union City, CA, USA). After midline skin incision, the surgery level was verified by using intraoperative plain X-rays. Posterior elements, including spinous processes, facet joints, lamina, and transverse processes, were exposed after muscle dissection. Pedicle screws (SMC; Taeyeon Medical, Wonju, Korea) were inserted bilaterally at the affected segments, including the upper and lower pedicles of the cage-inserting level under fluoroscopic guidance. The spinous process and lamina were removed using a Kerrison punch, rongeurs, and a high-speed drill. Bilateral facetectomies at the main level were performed using small rongeurs, osteotomes, and a high-speed drill. Inferior articular process was usually removed by osteotomes and superior articular process was removed by a high-speed drill to approach the intervertebral disc of the PTCIF level. It makes a safe access to the thoracic disc space without retraction of the spinal cord and saves corresponding pedicles. The pedicles around the intervertebral disc should be preserved to use a pedicle screw distractor in order to provide sufficient cage insertion space. After annulus incision, meticulous discectomy with endplate removal was performed (Fig. 2A). A shaver with a height of 8,10 , or $12 \mathrm{~mm}$, according to the disc height, was inserted directly into the disc space and rotated $90^{\circ}$ to remove disc material, identifying bony endplate under C-arm guidance (Fig. 2B). A pituitary forcep was then used to remove as
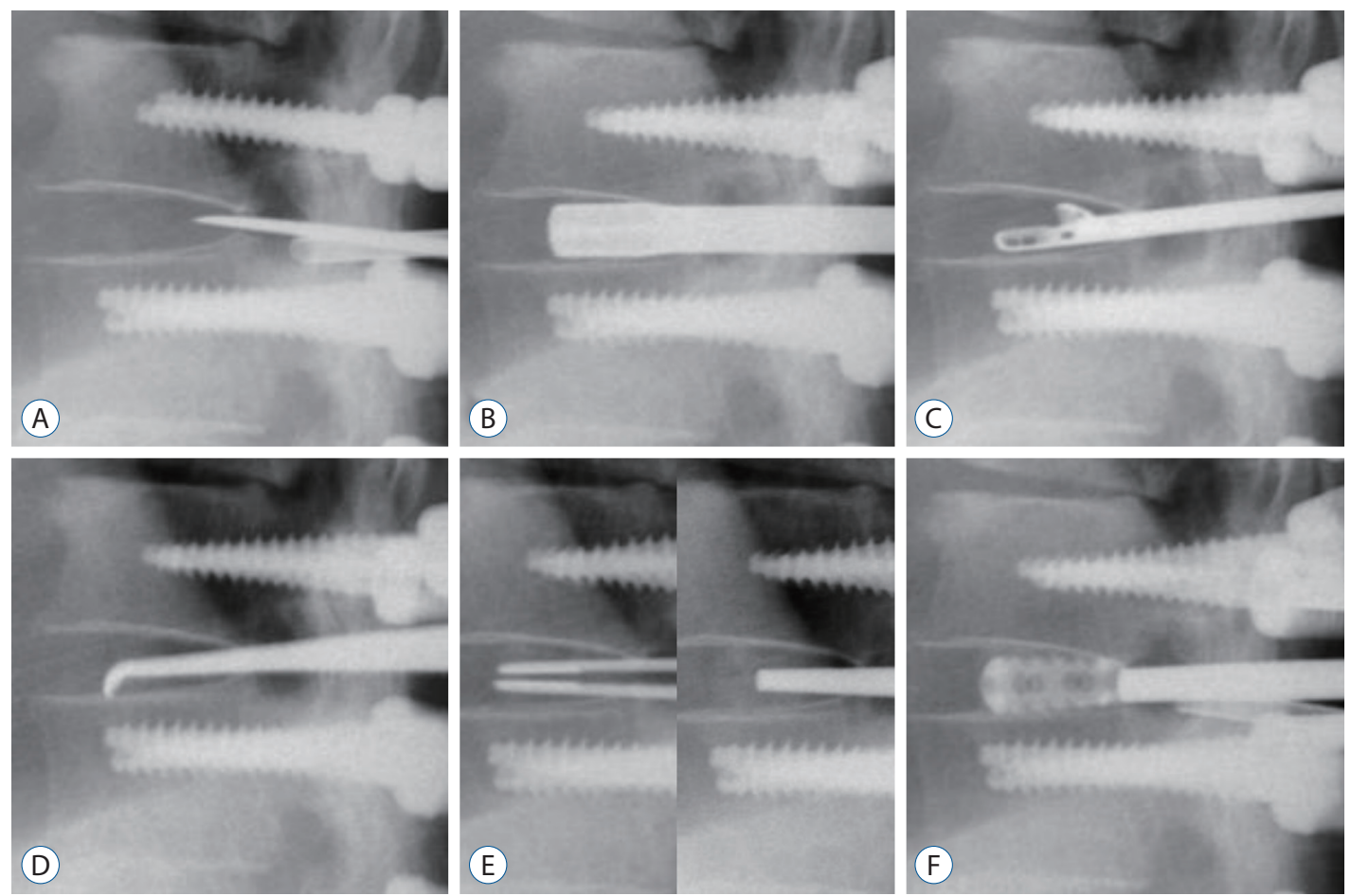

Fig. 2. Intraoperative C-arm images in posterior thoracic cage interbody fusion procedures. A : Discectomy was performed after the incision of the annulus using knife and sharp osteotome. B : A shaver was inserted and rotated to scrub the endplate. C : The disc material was removed using a pituitary forcep. D : The remnant disc material of the endplate was curetted with an angled curette. $\mathrm{E}$ : Autologous bone chips were inserted into the disc space and packed by a sharp impactor to enhance bone fusion before cage insertion. $\mathrm{F}$ : Thereafter, cages packed with autologous bone chips were inserted. 
much of the disc material as possible (Fig. 2C). Afterward, an angled curette was used to remove the remnant disc material of the endplate (Fig. 2D). Before cage insertion, autologous bone chips harvested from the removed lamina were inserted into the disc space to enhance bone fusion (Fig. 2E). Thereafter, cages (Neo-IC lumbar cage; U\&I Corporation, Uijeongbu, Korea) packed with autologous bone chips were inserted into the intervertebral space using a pedicle screw distractor for pedicle distraction (Fig. 2F). Subsequently the inserted cage was gently twisted $90^{\circ}$ before removing the pedicle screw distractor. Lateral spaces outside each cage in the interbody space were packed with additional local bone chips, followed by lateral rotation of the cages to provide capping and prevent bone chip migration into the spinal canal. During this procedure, keeping the pedicles intact is essential to distracting pedicles and obtaining sufficient space for cage insertion. The rods were connected and fixed to the screws while the disc space was compressed by a pedicle screw compressor. PLF at the PTCIF segment was not conducted. The cages were inserted carefully without any spinal cord retraction or manipulation. Cages were inserted unilaterally or bilaterally depending on the case.

\section{Conventional PLF technique}

Conventional PLF was conducted in the same manner as PTCIF until pedicle screw insertion and decompression. After wide decompression, rods were fixed to the screws and then, PLF was performed : bone chips were applied on fusion bed, which were obtained through extensive lateral muscle dissection to each transverse process and proximal ribs (Fig. 3). To perform PLF, sufficient fusion materials were needed. As a result, allograft or hydroxyapatite bone chips were used frequently along with autologous bone chips (Fig. 3B). Before paving with bone chips, the transverse process and proximal ribs were decorticated with a drill.

\section{Outcome evaluation}

Clinical data, including patient's age, sex, operative indications, presence of complications, and length of hospital stay were compared. The patient's pre- and postoperative neurological status was evaluated according to the American Spinal Injury Association impairment scale ${ }^{11)}$. Intraoperative data, including cage types, unilateral or bilateral cage insertion, estimated blood loss, and operation time were collected.

In each postoperative plain X-ray, the regional Cobb angle and the proximal junctional Cobb angle were measured. The regional Cobb angle was defined by measuring the angle between the tangential line to the cephalad endplate line of the upper instrumented vertebrae (UIV) and the tangential line to the caudal endplate line of the lower instrumented vertebrae $^{12)}$, and the proximal junctional Cobb angle was determined by measuring the angle between the tangential line to the cephalad endplate line of two supra-adjacent vertebrae above the UIV and tangential line to the caudal endplate line of the UIV (Fig. 4) $)^{8}$.

Bone fusion at the postoperative CT was defined as the for-
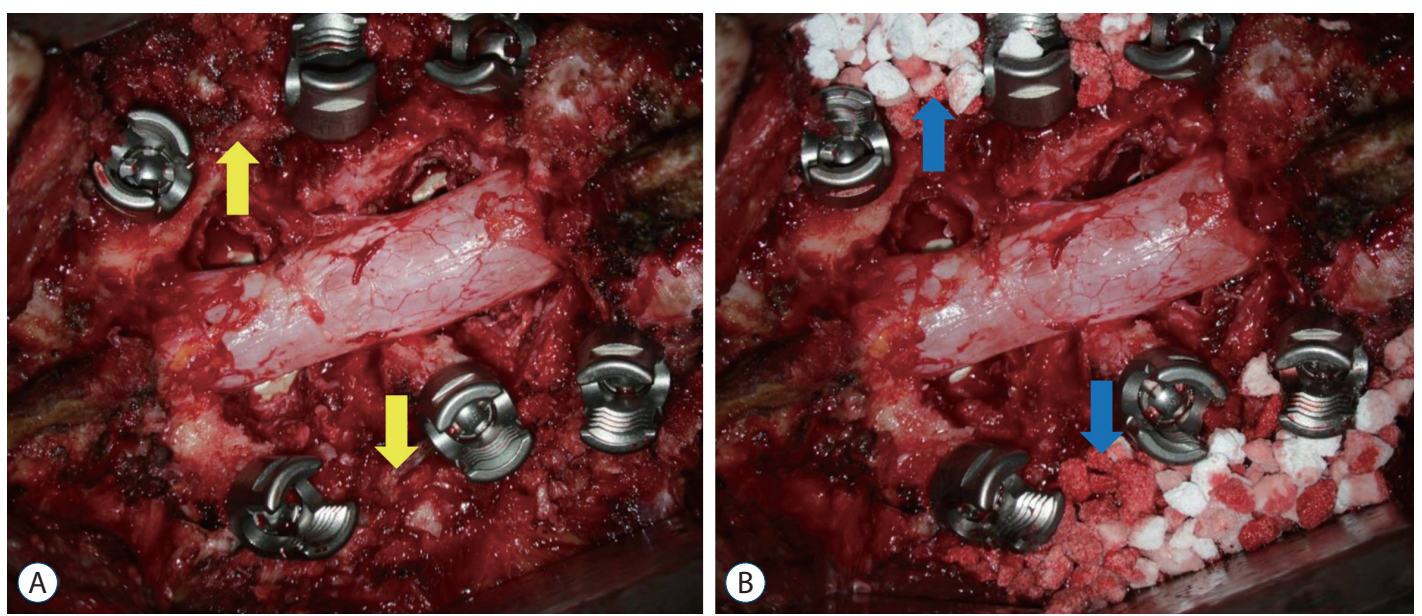

Fig. 3. Images of conventional posterolateral fusion after posterior screw fixation and laminectomy images at surgical field are shown. Bone chips were applied onto the fusion bed obtained through extensive lateral muscle dissection. A : The yellow arrows indicate the local autologous bone chips. B : Allograft or hydroxyapatite bone chips can be used along with autologous bone chips. The blue arrows indicate the hydroxyapatite bone chips. 


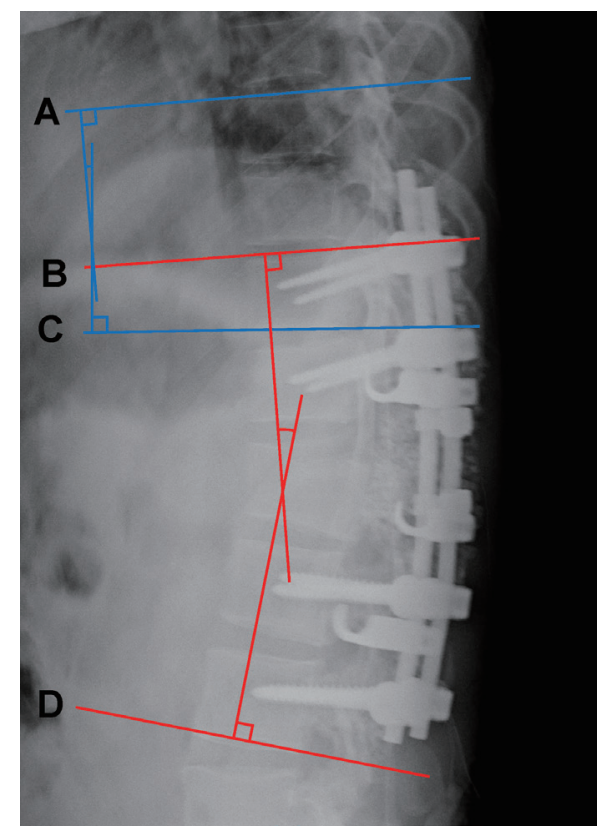

Fig. 4. Radiological measurement of the sagittal X-ray. The regional Cobb angle is the angle between the red tangential line to the cephalad endplate line of the upper instrumented vertebrae (UIV) (B) and the red tangential line to the caudal endplate line of the lower instrumented vertebrae (D). The proximal junctional Cobb angle is the angle between the blue tangential line to the cephalad endplate line of the two supraadjacent vertebrae above the UIV (A) and the blue tangential line to the caudal endplate line of the UIV (C). mation of bony continuity between the upper and lower end plates and circumjacent the cages in the PTCIF level ${ }^{22}$. On the contrary, solid bone fusion in conventional PLF was defined when a solid fusion mass was observed on simple X-ray. When there was an absence of a solid fusion mass but no evidence of halo around the implant and less than $2 \mathrm{~mm}$ of motion in the flexion-extension lateral radiographs, the bone fusion status was classified as indeterminate ${ }^{17,20)}$.

\section{Statistical analysis}

Quantitative data were presented as the mean \pm standard deviation unless otherwise indicated. Independent t-tests were used for continuous variables. Chi-square tests and Fisher's exact tests were used for categorical variables between the groups. A $p$-value $<0.05$ was considered statistically significant. Statistical analysis was performed using IBM SPSS software version 21 (IBM, SPSS, Chicago, IL, USA).

\section{RESULTS}

Of the 57 included patients, 30 (12 males and 18 females) received PTCIF and 27 (13 males and 14 females) received con-

Table 1. Baseline characteristics in patients of PTCIF and conventional PLF

\begin{tabular}{lccc}
\hline & PTCIF & Conventional PLF & $p$-value \\
\hline No. of patients & 30 & 27 & \\
Age at surgery (years) & $61.2 \pm 13.4$ & $58.2 \pm 17.6$ & 0.46 \\
Sex & & & 0.53 \\
$\quad$ Male & $12(40.0)$ & $13(48.1)$ & \\
$\quad$ Female & $18(60.0)$ & $14(51.9)$ & 0.94 \\
Operative indication & & & $2(7.4)$ \\
Burst fracture & $3(10.0)$ & $2(7.4)$ & \\
Fracture-dislocation & $2(6.7)$ & $1(3.7)$ & \\
Traumatic compression fracture & $1(3.3)$ & $13(48.1)$ & \\
Spinal stenosis & $15(50.0)$ & $2(25.9)$ & 0.23 \\
Spondylolisthesis & $5(16.7)$ & & \\
Herniated disc & $4(13.3)$ & $2(7.4)$ & \\
Anatomical level of offending lesion & & $12(44.4)$ & \\
Middle thoracic (T5-T8) & $0(0.0)$ & $13(60.1)$ & \\
Lower thoracic (T8-T11) & $12(40.0)$ & & \\
Thoracolumbar (T11-L2) &
\end{tabular}

Values are presented as mean \pm standard deviation or number (\%). PTCIF : posterior thoracic cage interbody fusion, PLF : posterolateral fusion 
Posterior Thoracic Cage Interbody Fusion I Shin HK, et al.

Table 2. Comparison of clinical parameters in patients of PTCIF and conventional PLF

\begin{tabular}{lccc}
\hline & PTCIF & Conventional PLF & p-value \\
\hline No. of patients & 30 & 27 & 0.46 \\
No. of levels fused* & $2.8 \pm 1.8$ & $3.1 \pm 1.7$ & 0.39 \\
No. of decompression levels & $2.4 \pm 1.4$ & $2.1 \pm 1.2$ & $<0.001$ \\
Operation time (minutes) & $452.0 \pm 127.3$ & $348.2 \pm 73.9$ & 0.01 \\
Length of hospital stay (days) & $14.7 \pm 6.8$ & $25.4 \pm 9.6$ & 0.56 \\
Estimated blood loss (mL) & $693.6 \pm 825.3$ & $579.6 \pm 619.9$ & 0.86 \\
Follow-up (months) & $35.6 \pm 19.9$ & $37.3 \pm 45.4$ & \\
Perioperative complications & & & $3(10.0)$ \\
$\quad$ Postoperative hematoma & $1(3.3)$ & $2(6.7)$ & \\
\multicolumn{1}{l}{ Wound infection } & $2(6.7)$ & & \\
\hline
\end{tabular}

Values are presented as mean \pm standard deviation or number (\%). *The levels of interbody cage insertion with laminectomy as well as upper and lower levels, which were fixed by pedicle screws without laminectomy and bone chips were applied on the lamina, were all included. PTCIF : posterior thoracic cage interbody fusion, PLF : posterolateral fusion

Table 3. Comparison of radiological parameters in patients of PTCIF and conventional PLF

\begin{tabular}{lccc}
\hline & PTCIF & Conventional PLF & p-value \\
\hline Regional Cobb angle $\left(^{\circ}\right.$ ) & & & 0.03 \\
Immediately after surgery, a & $6.9 \pm 4.8$ & $12.3 \pm 10.9$ & $<0.001$ \\
Last follow-up, $\beta$ & $8.3 \pm 3.4$ & $19.9 \pm 12.4$ & 0.003 \\
Difference between a and $\beta, \beta-a$ & $1.4 \pm 3.5$ & $7.6 \pm 9.1$ & 0.03 \\
Proximal junctional Cobb angle $\left(^{\circ}\right)$ & & $6.7 \pm 5.2$ & 0.06 \\
Immediately after surgery, a & $4.1 \pm 4.1$ & $8.8 \pm 6.1$ & 0.97 \\
Last follow-up, $\beta$ & $6.1 \pm 5.1$ & $2.1 \pm 5.3$ & 0.3 \\
Difference between a and $\beta, \beta-a$ & $2.0 \pm 4.2$ & & 0 \\
\hline
\end{tabular}

Values are presented as mean \pm standard deviation. PTCIF : posterior thoracic cage interbody fusion, PLF : posterolateral fusion

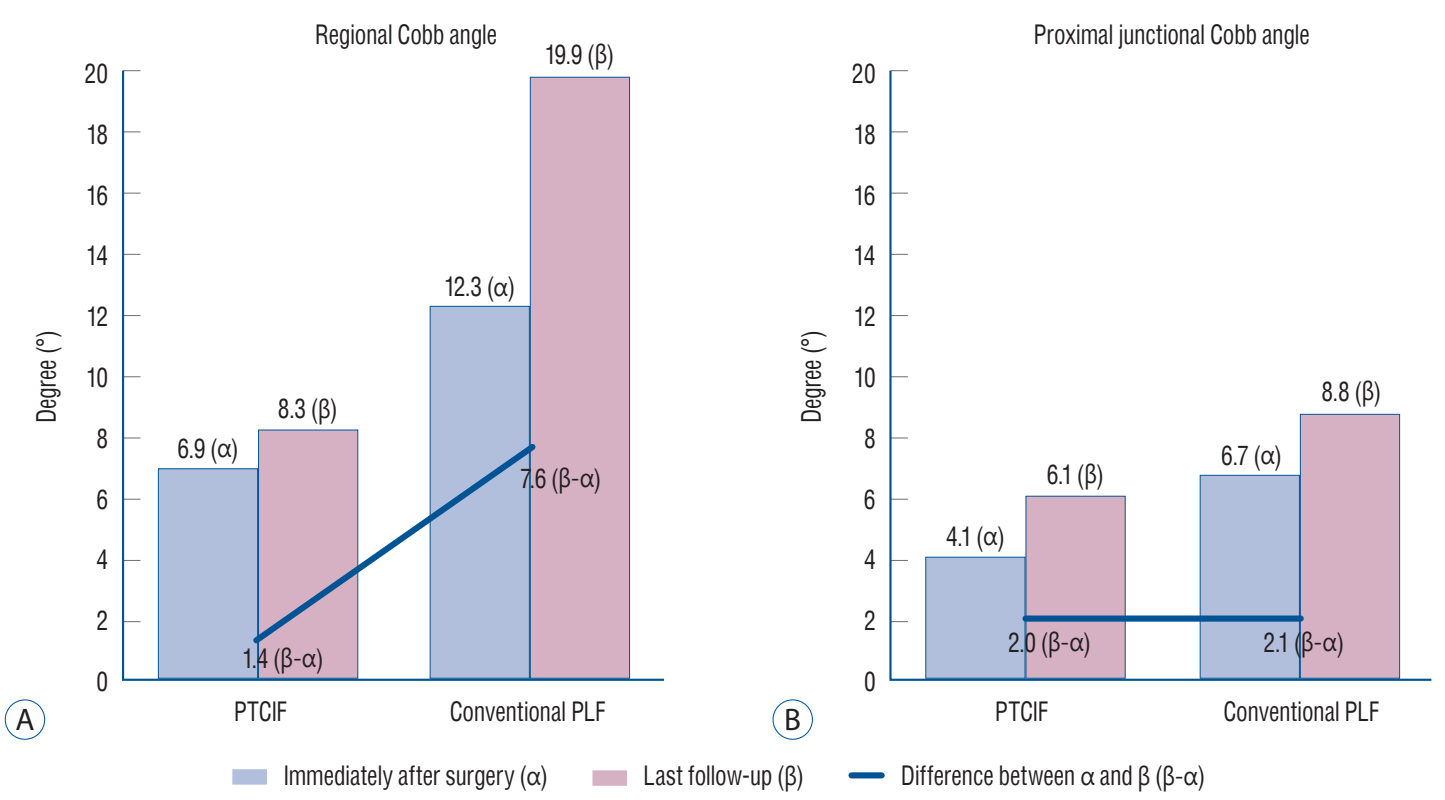

Fig. 5. The changes of radiological parameters of the two groups during follow-up. A : The mean difference in the regional Cobb angle immediately after surgery compared with that of the last follow-up was $1.4^{\circ}$ in posterior thoracic cage interbody fusion (PTCIF) and $7.6^{\circ}$ in conventional posterolateral fusion (PLF), which showed significant difference between the two groups $(p=0.003)$. $B$ : The mean difference in the proximal junctional Cobb angle immediately after surgery compared with that of the last follow-up was $2.0^{\circ}$ in PTCIF and $2.1^{\circ}$ in conventional PLF, which showed no significant difference ( $p=0.97$ ). 
ventional PLF. There was no difference between the groups regarding the age $(p=0.46)$, sex $(p=0.53)$, operative indication ( $p=0.94$ ), and anatomic level of offending lesion $(p=0.23)$ (Table 1). The numbers of levels fused $(p=0.46)$ and the numbers of decompression levels $(p=0.39)$ were not different between the groups. PTCIF was identified as needing longer operation times (PTCIF : 452.0 vs. conventional PLF : 348.2 minutes, $p<0.001)$, whereas the length of hospital stay was shorter than conventional PLF (PTCIF : 14.7 vs. conventional PLF : 25.4 days, $p=0.01$ ) with similar estimated blood losses (PTCIF : 693.6 vs. conventional PLF : $579.6 \mathrm{~mL}, p=0.56$ ). The mean durations of postoperative follow-up were not different (PTCIF : 35.6 vs. conventional PLF : 37.3 months, $p=0.86$ ) (Table 2 ).

Every patient showed functional improvement except one

Table 4. Characteristics of PTCIF surgery

\begin{tabular}{|c|c|}
\hline & Value \\
\hline \multicolumn{2}{|c|}{ Levels of PTCIF only $(n=30)^{*}$} \\
\hline \multicolumn{2}{|c|}{1 level (19 cases, 63.3\%) } \\
\hline T10-11 & $6(20.0)$ \\
\hline $\mathrm{T} 11-12$ & $7(23.3)$ \\
\hline $\mathrm{T} 12-\mathrm{L} 1$ & $5(16.7)$ \\
\hline L1-2 & $1(3.3)$ \\
\hline \multicolumn{2}{|c|}{2 level (9 cases, 30.0\%) } \\
\hline T8-9-10 & $1(3.3)$ \\
\hline T10-11-12 & $4(13.3)$ \\
\hline $\mathrm{T} 11-12-\mathrm{L} 1$ & $2(6.7)$ \\
\hline T12-L1-2 & $1(3.3)$ \\
\hline L1-2-3 & $1(3.3)$ \\
\hline \multicolumn{2}{|c|}{3 level (2 cases, 6.7\%) } \\
\hline T12-L1-2-3 & $1(3.3)$ \\
\hline T10-11, L1-2-3 & $1(3.3)$ \\
\hline \multicolumn{2}{|c|}{ Lateralities of cage $(n=43)$} \\
\hline Unilateral & $26(60.5)$ \\
\hline Bilateral & $17(39.5)$ \\
\hline \multicolumn{2}{|l|}{ Types of cage $(n=43)$} \\
\hline $8 \mathrm{~mm}, 0^{\circ}$ & $22(51.1)$ \\
\hline $8 \mathrm{~mm}, 4^{\circ}$ & $11(25.6)$ \\
\hline $10 \mathrm{~mm}, 0^{\circ}$ & $2(4.7)$ \\
\hline $10 \mathrm{~mm}, 4^{\circ}$ & $1(2.3)$ \\
\hline $10 \mathrm{~mm}, 8^{\circ}$ & $4(9.3)$ \\
\hline $12 \mathrm{~mm}, 4^{\circ}$ & $1(2.3)$ \\
\hline $12 \mathrm{~mm}, 8^{\circ}$ & $2(4.7)$ \\
\hline
\end{tabular}

Values are presented as number (\%). *Upper and lower levels, which were fixed by pedicle screws without laminectomy and bone chips were applied on the lamina, were excluded. PTCIF : posterior thoracic cage interbody fusion case of PTCIF. Postoperative hematoma occurred in one case in PTCIF and three cases in conventional PLF which required additional hematoma evacuation surgery in all cases. Superficial wound infection occurred in two cases in PTCIF and two cases in conventional PLF which were resolved with antibiotics (Table 2).

Radiological parameters are shown in Table 3. The mean difference in the regional Cobb angle immediately after surgery compared with that of the last follow-up was $1.4^{\circ}$ in PTCIF and $7.6^{\circ}$ in conventional PLF, which showed significant difference between the groups ( $p=0.003$ ) (Fig. $5 \mathrm{~A}$ ), whereas the mean difference in the proximal junctional Cobb angle immediately after surgery compared with that of the last follow-up was $2.0^{\circ}$ in PTCIF and $2.1^{\circ}$ in conventional PLF, which showed no significant difference ( $p=0.97$ ) (Fig. $5 \mathrm{~B}$ ).

Table 4 is the characteristics of PTCIF surgery. Of the patients who were treated by PTCIF, 1 level PTCIF was mostly performed on 19 patients (63.3\%), and 2 level PTCIF was performed on nine patients (30.0\%). One level PTCIF was mostly performed at T11-12 (seven among 19 cases) and 2 level PTCIF mostly performed at T10-11-12 (four among nine cases). Unilateral cages were inserted to 26 levels $(60.5 \%)$ and bilateral cages were inserted to 17 levels (39.5\%). Eight $\mathrm{mm}, 0^{\circ}$ cages were mostly used (22 level, 51.1\%) and $8 \mathrm{~mm}, 4^{\circ}$ cages were the second most commonly used (11 level, 25.6\%). Table 5 is the characteristics of conventional PLF surgery. Of the patients who were treated by conventional PLF, 4 level PLF was mostly performed on 11 patients (40.7\%)

Postoperative fusion status is shown in Table 6. In PTCIF, follow-up CT about 3-4 months after the operation revealed the formation of bony continuity within or circumjacent the cages between the upper and lower endplates at the PTCIF

Table 5. Characteristics of conventional PLF surgery

\begin{tabular}{lc}
\hline & Value \\
\hline Levels of conventional PLF $(n=27)$ & \\
1 level & $6(22.2)$ \\
2 level & $6(22.2)$ \\
3 level & $1(3.7)$ \\
4 level & $11(40.7)$ \\
5 level & $1(3.7)$ \\
6 level & $0(0.0)$ \\
7 level & $2(7.4)$ \\
\hline
\end{tabular}

Values are presented as number (\%). PLF : posterolateral fusion 
level in all cases, whereas detection of bone fusion is difficult in conventional PLF since measuring the connectivity of bony fragment is complex (Fig. 6A and B). In conventional PLF, it was not easy to determine the fusion status using the criteria

Table 6. Postoperative fusion status of PTCIF and conventional PLF surgery

\begin{tabular}{lcc}
\hline & PTCIF $(\mathbf{n}=\mathbf{3 0})$ & Conventional PLF $(\mathbf{n}=\mathbf{2 7})$ \\
\hline Solid fusion & $30(100.0)$ & $8(29.6)$ \\
Indeterminate & $0(0.0)$ & $19(70.4)$ \\
Non-fusion & $0(0.0)$ & $0(0.0)$ \\
\hline
\end{tabular}

Values are presented as number (\%). PTCIF : posterior thoracic cage interbody fusion, PLF : posterolateral fusion of fusion, since most cases were proved to be indeterminate status $(70.4 \%)$ which could be considered as clinical fusion (Fig. 6C and D). However, there was neither a definite non-fusion case nor a revision case due to pseudoarthrosis.

\section{DISCUSSION}

Conventional PLF, in lumbar spine, consists of pedicle screw fixation with bone graft application between the transverse processes and lateral aspects of the facet joints. In contrast, the PLIF technique is conducted by inserting a cage packed with bone chips after discectomy ${ }^{6}$. Many studies have
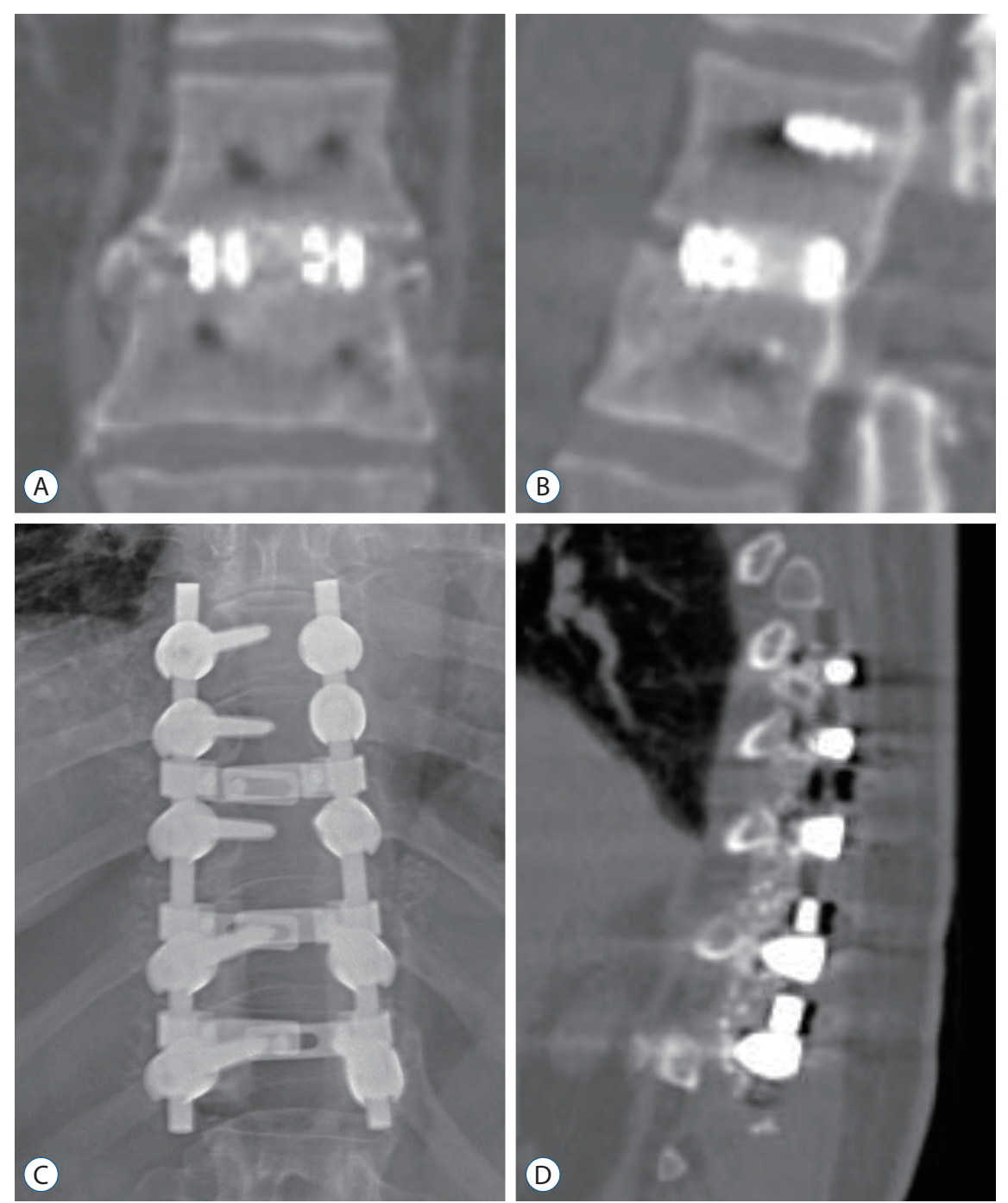

Fig. 6. Bony continuity within and circumjacent the cages between the upper and lower endplates are well identified in coronal (A) and sagittal (B) CT images 3 months after PTCIF at T12-L1, whereas it is complex to verify the bony continuity in simple spine X-ray (C) and sagittal CT image (D) 3 months after conventional PLF. PTCIF : posterior thoracic cage interbody fusion, PLF : posterolateral fusion, CT : computed tomography. 
compared these two approaches regarding technical demands and clinical outcomes; PLIF is superior in many aspects compared to the conventional PLF technique $e^{5,7,9,13)}$. Theoretically, the advantages of PLIF include anterior column support, indirect foraminal decompression, and restoration of lordosis ${ }^{23,24)}$.

In thoracic and TL spine, however, conventional PLF has been exclusively utilized for spinal fusion. It is important to consider the anatomical difference of the thoracic and TL spine and the lumbar spine. First, the lamina of the thoracic spine is narrower than that of the lumbar spine ${ }^{25)}$. Accordingly, more lateral dissection is necessary to expose bilateral rib head even in non-decompression surgery, which results in chronic postoperative back pain and muscle atrophy ${ }^{18)}$. In addition, it is technically more difficult to place sufficient bone chips in laminectomy surgery, as the fusion bed is not large enough due to the risk of bone chip migration onto the exposed spinal cord after laminectomy, causing spinal cord compression.

We hypothesized that PTCIF, in a similar manner with PLIF performed in lumbar segments, can be applied to the thoracic and TL spine. Not only does PTCIF help to restore the anterior and middle columns via cage insertion resulting in a better fusion rate, but it also can reduce the amount of bone graft required for successful fusion and avoids unnecessary iliac bone harvest ${ }^{1)}$. Another advantage of PTCIF is that there is no need to dissect muscle widely beyond the transverse processes. In our previous study, we identified that PLIF with pedicle screw fixation showed successful bone fusion at very high rates (100\% fusion rate $)^{21)}$, and in this present study, our results confirmed early successful bone fusion at the PTCIF sites in all patients, while the fusion rate of conventional PLF was reported to be $85 \%$ and $89.1 \%$ in lumbar and thoracic spine in other studies ${ }^{2,3}$. Furthermore, our results clarified that postoperative regional kyphotic change could be prevented by using the PTCIF technique through anterior column support, compared to conventional PLF, which showed regional kyphotic change.

The indication of trauma types for PTCIF were burst fracture, fracture-dislocation, and traumatic compression fracture. However, we don't think that PTCIF technique is applicable to entire cases of burst fracture. Regarding the indication of PTCIF for burst or compression fracture, the severity of comminution in the vertebral body might be important. The vertebral body should be preserved or minimally destructed and the removal of bony fragments encroaching the spinal canal is possible by posterior approach if we apply the PTCIF to these fracture cases. When vertebral body is showing severe body comminution with kyphotic deformity in burst fracture, the PTCIF could not be indicated : instead, reconstruction using expandable cage or titanium mesh cage should be performed for fusion after corpectomy in such cases. We can establish the precise indication criteria of PTCIF technique in trauma cases including burst fracture as well as compression fracture through further studies.

In thoracic and TL spine, the vertebral canal is smaller than the lumbar spine and spinal cord manipulation is challenging due to its high susceptibility to injury. There were a few published studies to perform thoracic interbody fusion through the posterior approach. Bransford et al. ${ }^{4)}$ performed a modified transfacet pedicle-sparing decompression and fusion on 18 patients diagnosed with herniated disc. Yamasaki et al. ${ }^{26)}$ reported the cases of 11 patients with herniated disc who were treated with the posterior thoracic interbody fusion. Their technique involved bilateral facetectomy with bone chips inserted into the intervertebral disc space, rather than cage insertion. However, we exclusively inserted cages filled with bone chips for superior fusion outcome and restoration of the disc height. The method reported here is distinct from the previously reported methods : it employs cages and spares the adjacent pedicles for insertion of the screws, resulting in short segment fixation.

Since over distraction of disc space in thoracic spine might be dangerous, to prevent cord injury from the manipulations during the surgery including over distraction, intraoperative evoked potential monitoring is always applied for this PTCIF technique. Even in the cases with $12 \mathrm{~mm}$ cage insertion, we did not distract excessively. Interbody cage size was decided according to the interbody disc space which was prepared with disc material and endplate removal using curette and interbody shaver. After disc material and endplate removal, the interbody height was evaluated by interbody shaver fitting tight, and then cage with same size of the interbody shaver was inserted with slight distraction of upper and lower pedicle screw. Twelve mm size cages were used only in fracture-dislocation cases and usually 8 or $10 \mathrm{~mm}$ size cages are appropriate in the degenerative thoracic spine disease as shown in Table 4. Even though 0 degree cages were usually used in PTCIF, lordotic cages (angled cages) were sometimes needed in disc 
spaces with concave shape or with partially destructed endplate by curettage. During this PTCIF procedure, careful disc space distraction should be always performed, especially in the motor evoked potential wave is negative due to trauma or severe paraparesis patients. Alternatively, when we cannot conduct safe and sufficient distraction of the disc space, narrowing of the disc space by pedicle screw compressor after cage insertion can acquire the same antipull-out strength.

Our study is limited in that it is retrospective and performed in a single institution. Also, the number of enrolled cases was small. Besides that, the follow-up period should be extended to verify its relation to delayed complications such as ASD.

\section{CONCLUSION}

PTCIF was found to be safe and achieve good outcomes for spinal cord decompression and bone fusion surgery. Our study showed that PTCIF elicits early and higher fusion rates as well as curvature maintenance compared to conventional PLF. PTCIF could be considered as an alternative procedure for posterior decompression and fusion surgery at the thoracic and TL spine.

\section{CONFLICTS OF INTEREST}

No potential conflict of interest relevant to this article was reported.

\section{INFORMED CONSENT}

This type of study does not require informed consent.

\section{AUTHOR CONTRIBUTIONS}

\author{
Conceptualization : SRJ \\ Data curation : HKS, MK, SKO, IC, DKS \\ Formal analysis : HKS \\ Funding acquisition : SRJ \\ Methodology : HKS, MK
}

Project administration : JHP, SWR, SRJ

Visualization : HKS

Writing - original draft : HKS, SRJ

Writing - review \& editing : SRJ

\section{ORCID}

Hong Kyung Shin https://orcid.org/0000-0001-8182-3321

Moinay Kim https://orcid.org/0000-0002-6443-7098

Sun Kyu Oh https://orcid.org/0000-0003-3496-1929

Il Choi https://orcid.org/0000-0003-0120-6564

Dong Kwang Seo https://orcid.org/0000-0002-0768-3743

Jin Hoon Park https://orcid.org/0000-0002-0903-3146

Sung Woo Roh https://orcid.org/0000-0001-6562-4154

Sang Ryong Jeon https://orcid.org/0000-0002-8340-7978

\section{- Acknowledgements}

This research was supported by a grant of the Korea Health Technology R\&D Project through the Korea Health Industry Development Institute (KHIDI), funded by the Ministry of Health \& Welfare, Republic of Korea (grant number: HI16C2188).

\section{References}

1. Agazzi S, Reverdin A, May D : Posterior lumbar interbody fusion with cages: an independent review of 71 cases. J Neurosurg 91 : 186-192, 1999

2. Alsaleh KA, Tougas CA, Roffey DM, Wai EK : Osteoconductive bone graft extenders in posterolateral thoracolumbar spinal fusion: a systematic review. Spine (Phila Pa 1976) 37 : E993-E1000, 2012

3. Bono CM, Lee CK : Critical analysis of trends in fusion for degenerative disc disease over the past 20 years: influence of technique on fusion rate and clinical outcome. Spine (Phila Pa 1976) 29 : 455-63; discussion Z5, 2004

4. Bransford R, Zhang F, Bellabarba C, Konodi M, Chapman JR : Early experience treating thoracic disc herniations using a modified transfacet pedicle-sparing decompression and fusion. J Neurosurg Spine 12 : 221-231, 2010

5. Cheng $L$, Nie $L$, Zhang $L$ : Posterior lumbar interbody fusion versus posterolateral fusion in spondylolisthesis: a prospective controlled study in the Han nationality. Int Orthop 33 : 1043-1047, 2009

6. Cloward RB : The treatment of ruptured lumbar intervertebral discs by vertebral body fusion. I. Indications, operative technique, after care. J 
Neurosurg $10: 154-168,1953$

7. Feng Y, Chen L, Gu Y, Zhang ZM, Yang HL, Tang TS : Restoration of the spinopelvic sagittal balance in isthmic spondylolisthesis: posterior lumbar interbody fusion may be better than posterolateral fusion. Spine J 15 : 1527-1535, 2015

8. Glattes RC, Bridwell KH, Lenke LG, Kim YJ, Rinella A, Edwards C 2nd : Proximal junctional kyphosis in adult spinal deformity following long instrumented posterior spinal fusion: incidence, outcomes, and risk factor analysis. Spine (Phila Pa 1976) 30 : 1643-1649, 2005

9. Kim KT, Lee SH, Lee YH, Bae SC, Suk KS : Clinical outcomes of 3 fusion methods through the posterior approach in the lumbar spine. Spine (Phila Pa 1976) 31 : 1351-1357; discussion 1358, 2006

10. Kim M, Oh SK, Choi I, Seo DK, Roh SW, Jeon SR : Clinical outcomes of posterior thoracic cage interbody fusion (PTCIF) to treat trauma and degenerative disease of the thoracic and thoracolumbar junctional spine. J Clin Neurosci 60 : 117-123, 2019

11. Kirshblum SC, Burns SP, Biering-Sorensen F, Donovan W, Graves DE, Jha $A$, et al. : International standards for neurological classification of spinal cord injury (revised 2011). J Spinal Cord Med 34 : 535-546, 2011

12. Lakshmanan P, Jones A, Mehta J, Ahuja S, Davies PR, Howes JP : Recurrence of kyphosis and its functional implications after surgical stabilization of dorsolumbar unstable burst fractures. Spine J 9 : 1003-1009, 2009

13. Liu XY, Qiu GX, Weng XS, Yu B, Wang YP : What is the optimum fusion technique for adult spondylolisthesis-PLIF or PLF or PLIF plus PLF? A meta-analysis from 17 comparative studies. Spine (Phila Pa 1976) 39 : 1887-1898, 2014

14. McDonnell MF, Glassman SD, Dimar JR 2nd, Puno RM, Johnson JR : Perioperative complications of anterior procedures on the spine. J Bone Joint Surg Am 78 : 839-847, 1996

15. Ohnishi K, Miyamoto K, Kanamori Y, Kodama H, Hosoe H, Shimizu K : Anterior decompression and fusion for multiple thoracic disc herniation.

J Bone Joint Surg Br 87 : 356-360, 2005

16. Okuyama K, Abe E, Suzuki T, Tamura Y, Chiba M, Sato K : Posterior lumbar interbody fusion: a retrospective study of complications after facet joint excision and pedicle screw fixation in 148 cases. Acta Orthop Scand $70: 329-334,1999$

17. Pearcy MJ, Tibrewal SB : Axial rotation and lateral bending in the normal lumbar spine measured by three-dimensional radiography. Spine (Phila Pa 1976) 9 : 582-587, 1984

18. Pourtaheri S, Issa K, Lord E, Ajiboye R, Drysch A, Hwang K, et al. : Paraspinal muscle atrophy after lumbar spine surgery. Orthopedics 39 : e209-e214, 2016

19. Ray CD : Threaded titanium cages for lumbar interbody fusions. Spine (Phila Pa 1976) 22 : 667-679; discussion 679-680, 1997

20. Sengupta DK, Truumees E, Patel CK, Kazmierczak C, Hughes B, Elders G, et al. : Outcome of local bone versus autogenous iliac crest bone graft in the instrumented posterolateral fusion of the lumbar spine. Spine (Phila Pa 1976) 31 : 985-991, 2006

21. Seo DK, Kim MJ, Roh SW, Jeon SR : Morphological analysis of interbody fusion following posterior lumbar interbody fusion with cages using computed tomography. Medicine (Baltimore) 96 : e7816, 2017

22. Shah RR, Mohammed S, Saifuddin A, Taylor BA : Comparison of plain radiographs with $\mathrm{CT}$ scan to evaluate interbody fusion following the use of titanium interbody cages and transpedicular instrumentation. Eur Spine J 12 : 378-385, 2003

23. Sudo H, Oda I, Abumi K, Ito M, Kotani Y, Minami A : Biomechanical study on the effect of five different lumbar reconstruction techniques on adjacent-level intradiscal pressure and lamina strain. J Neurosurg Spine 5 : 150-155, 2006

24. van Dijk M, Smit TH, Sugihara S, Burger EH, Wuisman PI : The effect of cage stiffness on the rate of lumbar interbody fusion: an in vivo model using poly(l-lactic Acid) and titanium cages. Spine (Phila Pa 1976) 27 : 682-688, 2002

25. Xu R, Burgar A, Ebraheim NA, Yeasting RA : The quantitative anatomy of the laminas of the spine. Spine (Phila Pa 1976) 24 : 107-113, 1999

26. Yamasaki R, Okuda S, Maeno T, Haku T, Iwasaki M, Oda T : Surgical outcomes of posterior thoracic interbody fusion for thoracic disc herniations. Eur Spine J 22 : 2496-2503, 2013 